\title{
Cannabis e humor
}

\section{Cannabis and mood}

\author{
Rafael Faria Sanches, ${ }^{1,2}$ João Mazzoncini de Azevedo Marques ${ }^{1}$ \\ 1 Departamento de Neurociências e Ciências do Comportamento, Faculdade de Medicina de Ribeirão Preto (FMRP), Universidade de \\ São Paulo (USP), Ribeirão Preto, SP, Brasil \\ 2 Instituto Nacional de Ciência e Tecnologia Translacional em Medicina (CNPq), Ribeirão Preto, SP, Brasil
}

\begin{abstract}
Resumo
Objetivo: Avaliar as relaçôes entre o uso agudo e crônico de cannabis e alterações do humor. Método: Os artigos foram selecionados por meio de busca eletrônica no indexador PubMed. Capítulos de livros e as listas de referências dos artigos selecionados também foram revisados. Resultados: Observam-se elevados índices de comorbidade entre abuso/ dependência de cannabis e transtornos afetivos em estudos transversais e em amostras clínicas. Estudos longitudinais indicam que, em longo prazo, o uso mais intenso de cannabis está relacionado com um risco maior de desenvolvimento de doença bipolar e, talvez, depressão maior em indivíduos inicialmente sem quadros afetivos; porém, os mesmos não encontraram maior risco de uso de cannabis entre aqueles com mania ou depressão sem esta comorbidade. Outra importante observação é que o uso de substâncias psicoativas em bipolares pode estar associado a uma série de características negativas, como dificuldade na recuperação dos sintomas afetivos, maior número de internações, piora na adesão ao tratamento, risco aumentado de suicídio, agressividade e a uma pobre resposta ao lítio. Tratamentos psicossociais e farmacológicos são indicados para o manejo da comorbidade entre cannabis e transtornos afetivos. Conclusão: As relaçôes entre o uso de cannabis e alterações do humor são observadas tanto epidemiologicamente quanto nos contextos clínicos.
\end{abstract}

Descritores: Cannabis; Depressão; Transtorno bipolar; Fatores de risco; Estudos transversais

\begin{abstract}
Objective: Evaluate the relationship between acute and chronic use of cannabis and mood changes. Method: Articles were selected by electronic search in PubMed. Chapters in books and reference lists of selected articles were also reviewed. As the research did not involve humans, there was no evaluation by a Research Ethics Committee. Results: High rates of comorbidity between uselabuseldependence of cannabis and affective disorders in longitudinal studies and in clinical samples were observed. Longitudinal studies indicate that, in long-term, the higher use of cannabis is associated with an increased risk of developing bipolar disorder, and probably, major depression in subjects initially without affective disorder, but was not found increased risk of cannabis use among those initially only with mania or depression. Another important observation is that substance abuse in bipolar patients may be associated with a number of negative characteristics, such as difficulty in recovering the affective symptoms, more hospitalizations, poor compliance with treatment, increased risk of suicide, aggression and a poor response to lithium. Psychosocial and pharmacological treatments are indicated for the management of comorbidity between cannabis and affective disorders. Conclusion: The relationship between cannabis use and mood changes are observed both in the epidemiological research and in the clinical settings.
\end{abstract}

Descriptors: Cannabis; Depression; Bipolar disorder; Risk factors; Cross sectional studies

\section{Introdução}

Os efeitos gerais do uso de cannabis sobre o humor e as relaçóes específicas com os transtornos afetivos (transtorno bipolar e depressão) são importantes epidemiologicamente, fornecendo informações significativas para a organização de serviços de saúde mental e para a clínica cotidiana.

De forma geral, pode-se considerar duas importantes relaçôes do uso de cannabis com o humor: 1) alterações do seu estado basal, no caso de usuários sem um quadro psiquiátrico estabelecido e 2) desencadeamento ou intensificação de sintomas de alterações psiquiátricas específicas.

Submetido: 15 Fevereiro 2010

Aceito: 8 Março 2010
Os artigos selecionados para presente revisão foram identificados por meio de busca eletrônica no banco de dados PubMed, identificando-se os estudos e revisōes da literatura sobre cannabis e humor. Capítulos de livros e as listas de referências dos artigos selecionados também foram revisados

\section{Alterações do estado basal do humor}

O efeito euforizante da cannabis provavelmente é o fator mais importante relacionado com a sua ampla difusão e uso. Usuários relatam frequentemente que essa sensação de euforia, 
relaxamento e despreocupação ("o barato") é a razão do uso da substância. Pequenas doses, quando usadas em grupo, com muita frequência induzem a um comportamento loquaz, risonho e alegre, muito semelhante àquele observado quando o álcool é utilizado "socialmente". Com o uso continuado, pode ocorrer tolerância a esse efeito, contribuindo para que alguns usuários passem a utilizar doses cada vez maiores e com mais frequência, desenvolvendo quadros de abuso e dependência ${ }^{1-4}$.

Por outro lado, uma parcela significativa dos usuários, principalmente os iniciantes, relata reaçôes disfóricas durante a intoxicação aguda, as quais podem incluir ansiedade importante (incluindo ataques de pânico com medo de morrer e de perder o controle), sensaçōes corporais desagradáveis, inquietação, despersonalização, desrealização e ideias paranoides. Pode ocorrer alternância entre sensações de euforia e disforia no mesmo indivíduo. Essa variabilidade de efeitos relatados provavelmente está relacionada com características específicas da substância (dose, modo de administração), do contexto em que é usada (em grupo ou sozinho, local de uso etc.) e do próprio usuário (características de personalidade, expectativas referentes ao uso $)^{1,2,4}$.

Alteraçóes psicológicas e comportamentais podem ser mantidas além da duração dos efeitos da intoxicação aguda por cannabis, incluindo sintomas afetivos como humor expansivo/ eufórico e aumento de autoestima/grandiosidade, além de sintomas psicóticos (delírios e alucinações), despersonalização e desrealização, os quais geralmente se resolvem em poucos dias. Esses quadros são normalmente relacionados ao uso de grandes quantidades da substância e podem estar se tornando mais comuns com o surgimento de preparaçōes cada vez mais potentes ${ }^{1-3,5,6}$.

A parada do uso crônico e intenso da substância pode levar ao aparecimento de sintomas como afeto disfórico (ansiedade, irritabilidade, tristeza), variações bruscas de humor, junto com outros sintomas psicológicos, comportamentais e somáticos. Até pouco tempo, persistia a controvérsia se estes sintomas constituem uma verdadeira síndrome de abstinência; porém, estudos recentes sugerem fortemente a existência desta ${ }^{7}$.

\section{Uso de cannabis e transtornos afetivos}

Diversos estudos têm encontrado uma associação importante entre o uso de cannabis e os quadros afetivos (transtorno afetivo bipolar, TAB, e depressão) nos serviços especializados de saúde mental, na assistência primária à saúde e na população como um todo. Inicialmente, discutiremos a relação entre o uso de cannabis e o transtorno bipolar e, depois, entre uso de cannabis e depressão, destacando os aspectos epidemiológicos e clínicos, os modelos etiológicos dessas comorbidades e as implicações quanto ao tratamento.

\section{Uso de cannabis e TAB}

\section{Aspectos epidemiológicos e clínicos}

O TAB apresenta a maior comorbidade com o abuso de substâncias entre todas as patologias do eixo I, sendo a cannabis a droga de escolha em cerca de $20 \%$ dos bipolares, índice que sobe quando se considera que dois terços deles já fizeram uso desta substância em algum momento de suas vidas ${ }^{8,9}$. Além disto, muitos estudos ${ }^{9-14}$ têm demonstrado que a comorbidade entre TAB e abuso/dependência à cannabis (A/DC) vai além do que seria esperado pelo acaso.

A associação destas duas patologias (TAB e A/DC) em um mesmo indivíduo pode ter um grande impacto na vida deste, como o observado em vários estudos. Sonne et al. e Feinman \& Dunner encontraram que o aparecimento precoce do TAB aumentaria a probabilidade de um uso posterior de drogas (principalmente álcool e maconha) $)^{15,16}$. No entanto, Strakowski et al. consideraram que, independentemente do que ocorre primeiro, a comorbidade entre cannabis e TAB piorava o prognóstico de pacientes bipolares, quando comparados aos mesmos, porém sem uso de cannabis $^{17}$. Em outro estudo, Henquet et al. acompanharam 4.815 sujeitos por um período de três anos, no qual foi verificado que o uso de cannabis no início do estudo aumentava significativamente o risco de aparecimento de sintomas maníacos durante o período de seguimento ${ }^{18}$. Os dados foram corrigidos para idade, sexo, nível educacional, etnia, estado civil, neuroticismo, sintomas depressivos e maníacos no período "baseline". Esta associação foi ainda independente da prevalência e incidência de sintomas psicóticos. Neste estudo não foi observado o inverso, ou seja, a presença de sintomas maníacos no baseline não aumentou o risco de uso da maconha no período de seguimento.

De um modo geral, parece que o uso de substâncias psicoativas em bipolares pode estar associado a uma série de características negativas, como dificuldade na recuperação dos sintomas afetivos, maior número de internações, piora na adesão ao tratamento, risco aumentado de suicídio, agressividade e a uma pobre resposta ao lítio ${ }^{13,19-23}$. No estudo de van Rossum et al., no qual mais de três mil sujeitos foram acompanhados por um período de um ano, os prejuízos clínicos foram bem mais evidentes do que os prejuízos sociais $^{20}$. Além disso, os bipolares que fizeram uso de cannabis tiveram maiores índices de uso de outras substâncias ilícitas. Assim, apesar do claro prejuízo em diversos aspectos clínicos que o uso da cannabis provoca em pacientes bipolares, a relativa preservação de alguns aspectos do funcionamento social pode desencorajar a busca por tratamento de modo precoce e, assim, piorar o seu prognóstico.

Outra questão importante nesta comorbidade é saber se o uso de cannabis aumenta o número de recaídas nestes pacientes. Strakowski et al. realizaram estudo prospectivo com um ano de seguimento e observaram que o uso da cannabis não está tão diretamente relacionado a recaídas (21\% continuaram o uso de cannabis e não recaíram neste período), como ocorre com o uso do álcool ${ }^{24}$. Este mesmo autor e seus colaboradores também encontraram uma associação entre uso de cannabis e mania, enquanto que a depressão foi associada ao uso de álcool ${ }^{13}$. No estudo de Feinman e Dunner, observou-se que os bipolares com co-ocorrência de problemas relacionados a álcool e drogas apresentavam mais ciclagem do que os bipolares "puros", talvez pela ação das drogas aumentando a labilidade do humor nestes pacientes ou pelo fato de que indivíduos com ciclagem rápida apresentam maior propensão ao uso de álcool e maconha ${ }^{16}$. 


\section{Modelos etiológicos para a associação entre uso de cannabis e TAB}

Alguns autores têm considerado o TAB como um transtorno com mais características biológicas quando comparado aos quadros depressivos e ansiosos ${ }^{25}$, facilitando, em parte, a investigação de teorias que possam explicar a co-ocorrência entre TAB e problemas relacionados a drogas. Os principais modelos para as possíveis explicações para a alta comorbidade entre estas duas patologias são: 1) fatores comuns; 2) uso secundário de substâncias; 3) desordens psiquiátricas secundárias; e, finalmente, 4) modelos bidirecionais.

No primeiro modelo haveria, por exemplo, uma vulnerabilidade genética ou presença de traços antissociais que seriam compartilhados tanto pelo TAB quanto pela dependência a drogas. No segundo modelo, o próprio TAB aumentaria a chance da ocorrência de uso de drogas. O terceiro modelo seria o oposto do segundo, ou seja, o uso de drogas levaria ao aparecimento de TAB em indivíduos não suscetíveis. No quarto e último modelo, tanto o TAB quanto o uso de drogas aumentaria a chance de ocorrência do outro transtorno. A seguir, serão apresentadas discussões relativas a cada um destes modelos.

\section{Fatores comuns}

Dentre os possíveis fatores comuns do TAB e do A/DC, merecem atenção os fatores genéticos, o transtorno de hiperatividade e déficit de atenção (TDAH), além traços de personalidade impulsiva e patologias específicas do eixo II.

Estudos em famílias de indivíduos bipolares, dependentes químicos e estudos com gêmeos mostram que fatores genéticos podem contribuir para o desenvolvimento de TAB e de A/DC. A questão é se a vulnerabilidade genética para um transtorno poderia facilitar o aparecimento do outro ${ }^{25,26}$.

Um dos fatores comuns a estes dois transtornos pode ser o transtorno de personalidade antissocial (TPAS). Estudos têm demonstrado que o TPAS e os transtornos de conduta na criança são fortemente associados ao uso de drogas na idade adulta e doenças psiquiátricas do eixo $\mathrm{I}^{10,27,28}$. Um fato que precisa ser considerado em futuros estudos refere-se à possibilidade de que o uso de drogas possa causar ou ser acompanhado de comportamentos antissociais, levando a um diagnóstico errôneo de TPAS $^{25}$.

Outra característica comum que pode estar envolvida na comorbidade $\mathrm{TAB}$ e $\mathrm{A} / \mathrm{DC}$ é a impulsividade enquanto traço do indivíduo. Neste sentido, há estudos que demonstram níveis aumentados de impulsividade basal (fora dos episódios de uso de substância e entre os episódios de mania), sendo que nos indivíduos comórbidos estes níveis são ainda maiores ${ }^{29,30}$. Distúrbios como o transtorno de personalidade borderline e o TDAH, que apresentam a impulsividade como uma de suas características, também são comuns tanto no TAB quanto nos indivíduos com problemas de $\mathrm{A} / \mathrm{DC}^{31,32}$.

\section{Uso secundário de substâncias}

Neste modelo, o estímulo para o uso de cannabis seria reforçado pela presença de um quadro bipolar prévio. No estudo de 20 anos de seguimento, de Merikangas et al., a presença de sintomas maniatiformes (abaixo da linha de corte para o transtorno bipolar) foi associado a um aumento no risco de desenvolvimento de abuso e dependência ao álcool, aos benzodiazepínicos e à cannabis ${ }^{33}$. Neste mesmo sentido, talvez o uso da cannabis possa ocorrer em decorrência de um pródromo do $\mathrm{TAB}$, no qual a presença de oscilações bruscas do humor, impulsividade e julgamento crítico da realidade prejudicado predisporiam ao uso da cannabis ${ }^{13,34}$.

Outra possibilidade para o aumento da comorbidade entre o $\mathrm{TAB}$ e o A/DC poderia ser decorrente de uma hipersensibilidade a substâncias ilícitas. A hipótese segue o modelo proposto para a esquizofrenia e, embora não possa se estender diretamente ao transtorno bipolar, o mesmo raciocínio pode ser válido. De acordo com este modelo, indivíduos com vulnerabilidade psicobiológica, determinada pela combinação de fatores genéticos e eventos ambientais precoces (período perinatal), reagem negativamente com estressores ambientais e desenvolvem o transtorno psiquiátrico, esquizofrenia ou TAB. Nesta situação, as medicaçōes psicotrópicas diminuiriam a vulnerabilidade, enquanto que as drogas poderiam aumentar esta vulnerabilidade ${ }^{25}$.

No modelo da automedicação, os pacientes procurariam a droga numa tentativa de alívio de vários sintomas do TAB, como por exemplo, a disforia ${ }^{25,35}$. Esta pode ser entendida como um dos sintomas-chave para o uso secundário de drogas, incluindo a cannabis. Sabe-se que pessoas bipolares são propensas a frequentes episódios de disforia, assim sendo, é sensato imaginar que esta sensação de mal-estar leve o indivíduo a procurar substâncias psicoativas. Em um primeiro momento, haveria alívio dos sintomas, até a instalação da adição a drogas, que então passaria a piorar o estado mental do paciente. Muitos estudos suportam a hipótese de que bipolares apresentam altos índices de disforia ${ }^{25}$, embora outros mostrem o contrário ${ }^{36,37}$.

Propõe-se também um modelo no qual múltiplos fatores de risco frequentemente ocasionados pelo transtorno mental - como isolamento social, pouca habilidade interpessoal, dificuldades cognitivas e/ou escolares, pobreza, falta de estrutura nas atividades diárias, associação com "turmas desviantes" - poderia levar ao uso secundário de drogas em pacientes bipolares. Esta ideia é reforçada pela observação de que alguns pacientes poderiam ter a sensação de uma melhora no convívio social ao fazerem uso de maconha ${ }^{20,25}$.

Como os estudos que investigam diretamente esta condição são escassos, não se pode confirmar esta teoria. Além disso, alguns autores ${ }^{13}$ relatam que, na maioria dos casos, o uso de drogas precede o aparecimento do $\mathrm{TAB}$, evidência contrária à hipótese de $\mathrm{A} / \mathrm{DC}$ secundária ao TAB.

\section{Desordens psiquiátricas secundárias}

Baseando-se na hipótese das catecolaminas do transtorno bipolar, pode-se supor que drogas que tenham ação em neurotransmissores são potencialmente relacionadas ao $\mathrm{TAB}^{25}$. Evidências como as mostradas por D'Souza et al. ${ }^{38}$ e Murray et al. ${ }^{39}$, de que o tetraidrocanabinol (THC) aumenta a atividade 
dopaminérgica mesolímbica e também os níveis glutamatérgicos no córtex pré-frontal podem estar relacionadas ao desenvolvimento de sintomas maniatiformes, uma vez que estas mesmas alterações são observadas na mania ${ }^{40-43}$.

Modelos animais ("kindling" e sensibilização comportamental) ${ }^{44}$ têm demonstrado ação sensibilizadora das drogas psicomiméticas sobre respostas eletrofisiológicas e comportamentais. Estes modelos podem estar associados ao mecanismo pelo qual as drogas de abuso precipitam o aparecimento de um quadro de esquizofrenia ou $\mathrm{TAB}^{45,46}$.

Os principais estudos nesta área não apresentam resultados unânimes, porém, a maioria deles tem indicado que o uso em idade precoce e regular de cannabis está associado a um risco aumentado de aparecimento de ideação/tentativa de suicídio, de sintomas maníacos ou até mesmo de um quadro afetivo ${ }^{18,36,47}$. No trabalho de Laar et al., o risco de desenvolvimento de TAB foi bastante expressivo, com aumento no risco de até cinco vezes mais chances de desenvolvimento de $\mathrm{TAB}$ em usuários regulares de maconha ${ }^{48}$.

\section{Modelos bidirecionais}

Os modelos bidirecionais sugerem interaçôes dos efeitos do TAB e do uso de drogas contribuindo para o aumento da comorbidade. Por exemplo, o uso de drogas poderia desencadear o aparecimento do $\mathrm{TAB}$ em indivíduos com predisposição genética. $\mathrm{O}$ TAB seria subsequentemente mantido pelo uso contínuo da droga por questôes culturais. Este modelo carece de evidências sólidas e de um número maior de estudos que possam testá-lo. Por hora, ele permanece no campo das hipóteses ${ }^{25}$.

Tratamento na comorbidade uso/abuso/dependência de cannabis e TAB

Há poucos estudos na literatura que delineiam as bases do tratamento de pacientes com comorbidade entre TAB e A/DC. Porém, independentemente da escolha medicamentosa, o suporte psicoterápico tanto para o paciente quanto para seus familiares é de suma importância. Neste sentido, destacam-se três tipos de terapias, que têm sido mais bem avaliadas neste grupo de pacientes: abordagens baseadas em entrevista motivacional, em terapias cognitivo-comportamentais e terapias comportamentais baseadas em manejo de contingências (fornecimento de incentivos para pacientes que realizam determinados comportamentos, como abstinência, por exemplo $)^{49}$. O trabalho conjunto com grupos de autoajuda e outras fontes de apoio na comunidade para o paciente é frequentemente possível e desejável, melhorando os resultados do tratamento ${ }^{50}$. $\mathrm{O}$ foco principal deve ser o controle (abstinência total, se possível) do uso de drogas e a estabilização do humor. Sabe-se que a interrupção do uso de drogas já pode, por si só, melhorar o quadro afetivo do paciente ${ }^{51,52}$.

O divalproato sódico e a carbamazepina são medicações com resultados satisfatórios em pacientes com TAB e abuso de substâncias, porém são medicações com risco de dano hepático; assim, devem ser usadas com cautela e com avaliação clínica rigorosa, pois pacientes com esta comorbidade apresentam, com frequência, hepatite viral ou alcoólica ${ }^{52}$.
O lítio apresenta bons resultados em alguns estudos, mas não em outros. Seu uso necessita de controle laboratorial rigoroso e apresenta risco significativo de intoxicação e/ou interações clinicamente significativas com outras substâncias ${ }^{52}$. Além disso, Weiss et al. relataram maior adesão com o valproato quando comparado ao lítio em bipolares com A/DC ${ }^{53}$.

$\mathrm{O}$ uso de antipsicóticos típicos pode estar relacionado a um aumento da procura por drogas ilícitas nestes pacientes. Entretanto, há relatos de que o uso da clozapina, um antipsicótico atípico, está associado a reduções no consumo de substâncias psicoativas $^{22}$. A quetiapina, outro medicamento atípico, mostrouse eficaz na redução média do consumo de maconha de $35 \mathrm{~g}$ por semana para apenas $1 \mathrm{~g} /$ semana em oito pacientes estudados por Potvin et al..$^{54}$.

Importante observação foi relatada no estudo de Strakowski et al., que após um período de internação por um quadro de mania, há uma janela na qual estes pacientes permanecem em abstinência à maconha, e sugerem, desta forma, intervenções incisivas e precoces neste período a fim de manter estes pacientes em abstinência à droga, o que se torna um desafio, visto que a maioria dos serviços públicos não têm por hábito agendar retornos precoces a pacientes egressos de internaçôes integrais ${ }^{17}$.

\section{Possíveis efeitos benéficos da cannabis sobre o TAB}

Discute-se a possibilidade de que as propriedades neurofarmacológicas dos canabinoides poderiam ajudar no tratamento de sintomas depressivos e/ou maníacos. Os canabinoides encontrados na planta da maconha poderiam exercer este efeito terapêutico por meio de suas ações anticonvulsivantes (possível ação estabilizadora) e protetoras contra a neurotoxicidade glutamatérgica ${ }^{8,35,55}$. Alguns poucos relatos de caso favorecem esta ideia, como as descrições de Grinspoon e Bakalar, que citaram cinco pacientes que apresentavam melhora dos sintomas maníacos quando faziam uso de maconha, inclusive com descriçôes de que ainda que o lítio pudesse também equilibrar o paciente, este não proporcionava a sensação de bem-estar que a cannabis causava ${ }^{55}$.

Gruber et al., por sua vez, relataram cinco casos de alívio de sintomas depressivos após o uso de cannabis, com eficácia superior ao uso de antidepressivos convencionais ${ }^{56}$. Estes possíveis efeitos terapêuticos são justificados pela literatura como decorrentes de certas propriedades farmacológicas tanto do THC quanto do canabidiol (CBD) - entre elas, pode-se citar a ação anticonvulsivante, ansiolítica e antipsicótica do CBD e o efeito antidepressivo do $\mathrm{THC}^{35,56-59}$.

Desta forma, a combinação de vários efeitos produzidos pelo THC (ação ansiolítica e antidepressiva) e pelo CBD (ação antipsicótica, anticonvulsivante e neuroprotetora) poderia apresentar ação terapêutica em pacientes bipolares ${ }^{60,61}$. No entanto, evidências iniciais do uso do CBD em modelos animais e no tratamento de episódio maníaco não confirmaram o efeito terapêutico desse canabinoide ${ }^{62,63}$. Assim, há que se ter em mente que estas "possíveis" ações terapêuticas proporcionadas pela maconha necessitam de comprovação sistemática e como 
os estudos até o momento mostram mais riscos da associação da cannabis no TAB do que benefícios, conclui-se que o uso da cannabis como adjuvante no tratamento do $\mathrm{TAB}$ deve ser restrito aos ambientes de pesquisa, exclusivamente ${ }^{17}$.

\section{Uso de cannabis e depressão}

\section{Aspectos epidemiológicos e clínicos}

Há décadas existem relatos de casos de desenvolvimento de sintomas depressivos relacionados ao uso de cannabis. No entanto, como já citado anteriormente, também foram descritos casos nos quais ocorreu o desenvolvimento de sintomas maníacos após o uso e também da cannabis sendo usada como antidepressivo ou estabilizador do humor ${ }^{36,56-58}$.

Estudos sobre as relações entre uso de cannabis e depressão em populações realizando tratamento psiquiátrico sugerem que a presença de abuso/dependência está relacionada com um maior número de episódios depressivos ao longo da vida e que o tratamento da dependência comórbida com depressão maior está associado a um pior resultado no sexo masculino ${ }^{36,64}$.

Estudos de prevalência realizados com amostras não representativas da população em geral (entre adolescentes, jovens adultos, universitários e pacientes de serviços de cuidados primários) encontraram resultados conflitantes entre si, difíceis de interpretar em conjunto pelas diferentes metodologias e definiçōes usadas (p.ex. quanto ao que é considerado usuário pesado ou ocasional), além de envolverem amostras pequenas que frequentemente não eram representativas da população específica estudada ${ }^{36}$.

Desde a publicação dos resultados do Epidemiologic Catchment Área (ECA) ${ }^{9}$, vêm sendo descritos estudos envolvendo amostras representativas da população em geral, alguns dos quais avaliaram as associações transversais entre o uso de cannabis e depressão.

Alguns desses estudos descreveram a incidência de depressão maior ou transtornos afetivos em usuários de cannabis comparada com a incidência nos adultos em geral ou em adolescentes e adultos jovens. Dois estudos norte-americanos abordaram a questão: um deles encontrou, na população adulta, um risco 6,4 vezes maior de apresentar depressão entre aquelas pessoas que sofriam de abuso ou dependência de cannabis $^{65}$ e o outro estudo encontrou um risco 3,4 vezes maior ${ }^{66}$. Na Austrália, encontrou-se que os usuários de cannabis apresentavam um risco maior que a população em geral de apresentarem transtornos afetivos, com a prevalência desses transtornos chegando a $14 \%$ entre os dependentes de cannabis em comparação com os não usuários ${ }^{67}$.

Outros estudos em amostras representativas de adolescentes e jovens adultos também encontraram resultados semelhantes. Um trabalho australiano encontrou entre os adolescentes que já tinham usado cannabis uma chance três vezes maior de desenvolver depressão em comparação com aqueles que nunca haviam usado ${ }^{68}$; um estudo neozelandês encontrou um risco progressivamente aumentado de depressão conforme o aumento da frequência do uso de cannabis ${ }^{69}$.

Quanto à incidência de uso frequente de cannabis ou A/DC entre os indivíduos deprimidos, um dos estudos norte-americanos já citados encontrou que a chance de um adulto apresentar dependência de cannabis era 2,4 vezes maior entre aqueles que apresentaram depressão maior em relação à população em geral ${ }^{66}$. Um estudo europeu encontrou um risco 2,3 vezes maior de uso semanal de maconha entre adultos jovens com depressão em comparação com aqueles não deprimidos ${ }^{70}$.

\section{Modelos etiológicos para a associação entre uso de cannabis e depressão}

A associação entre uso de cannabis e depressão, como no caso da associação com transtorno bipolar, também pode ser explicada por basicamente quatro modelos: 1) existem fatores comuns que causam tanto depressão como uso de cannabis; 2) depressão é causa do uso de cannabis; 3) o uso de cannabis é causa de depressão; e 4) modelos bidirecionais. Provavelmente, a melhor maneira de avaliar essas possibilidades é por meio de estudos longitudinais ${ }^{3,36,71-73}$.

Assim, estudos longitudinais que avaliaram se a presença de depressão na adolescência estava relacionada com um risco aumentado de usar de cannabis posteriormente não encontraram associaçōes significativas ${ }^{36,47,74,75}$.

Uma revisão sistemática ${ }^{76}$ publicada em 2007 avaliou 10 estudos que examinaram a relação entre o uso de cannabis na adolescência e/ou início da vida adulta e o diagnóstico de depressão posteriormente (feito por meio de entrevistas estruturadas ou com o uso de questionários validados). Cinco desses estudos ${ }^{47,69,77-79}$ encontraram associações significativas mesmo após ajustamento estatístico considerando outras variáveis. Os outros estudos encontraram associações não significativas ou não encontraram associação de todo ${ }^{74,80-83}$. No conjunto de estudos (cinco no total) que avaliaram a exposição à cannabis por meio de medidas de frequência - o qual foi considerado adequado para metanálise pelos autores -, quatro descreveram uma associação tipo doseresposta entre frequência de uso de cannabis e risco de desenvolver depressão; encontrou-se um risco relativo de $1,49^{76}$. Os autores optaram por não realizar uma metanálise dos 10 estudos devido à heterogeneidade das medidas de exposição à cannabis (que incluíam qualquer uso, frequência de uso e abuso e dependência $)^{76}$.

Em outros três estudos - publicados posteriormente à confecção desta metanálise - foram descritos tanto resultados apontando para não associação entre uso de cannabis e depressão ${ }^{84,85}$ como para associação significativa ${ }^{48}$.

Então, podemos considerar que - tanto pelo conjunto dos resultados como pela qualidade metodológica insatisfatória de vários estudos - não está definido claramente se o uso de cannabis implica em risco aumentado de depressão.

Caso esse risco aumentado realmente ocorra, as razōes para isso podem estar relacionadas com vários fatores. Não parece que ações da cannabis em sistemas neurotransmissores estejam relacionadas com a facilitação da instalação de quadros depressivos. Provavelmente, os efeitos do uso de cannabis relacionados com o aumento do risco de depressão são mediados socialmente, pois existem evidências de que o uso regular e/ou precoce dessa substância possa facilitar situaçōes como desemprego, dificuldade de acesso à educação e envolvimento em crimes. Finalmente, 
ainda não é possível descartar completamente que fatores comuns relacionados à família e/ou ao meio social possam ser a causa da associação $^{36}$.

Para esclarecer essas questões são necessários mais estudos longitudinais populacionais e entre gêmeos discordantes para uso e/ou abuso e/ou dependência de cannabis e depressão ${ }^{36,74}$.

\section{Tratamento da comorbidade uso/abuso/dependência de cannabis e depressão}

Como já referido quanto ao $\mathrm{TAB}$, existem poucos estudos abordando especificamente o tratamento da comorbidade depressão e uso/abuso/dependência de substâncias psicoativas.

As terapias baseadas em entrevista motivacional, cognitivocomportamentais e de manejo de contingências também são úteis para tratar essa comorbidade ${ }^{49}$. A entrevista motivacional especialmente tem demonstrado bons resultados no tratamento da dependência de cannabis em estudo multicêntrico ${ }^{86}$. Quanto ao tratamento medicamentoso, apenas um estudo randomizado duplo-cego pesquisando a resposta a inibidores seletivos da recaptação de serotonina incluiu adolescentes com abuso e dependência de cannabis, encontrando que essas medicações são eficazes para tratar depressão nesta população. Um achado interessante do mesmo estudo foi o alto índice de resposta ao tratamento de depressão com terapia cognitivo-comportamental associada a placebo, mostrando novamente a importância das terapias não farmacológicas ${ }^{87}$.

\section{Conclusão}

Assim como para psicose ${ }^{88}$ e ansiedade ${ }^{89}$, esta revisão reforça a necessidade de investigação cuidadosa de presença concomitante de uso de maconha em pacientes com transtorno bipolar e depressão, bem como a possível presença de transtornos afetivos em indivíduos diagnosticados como usuários dessa substância. Isto pode proporcionar uma abordagem mais adequada, com chances de melhor prognóstico. $\mathrm{O}$ tratamento deve abranger as duas condiçóes e as outras necessidades individuais de cada paciente.

Financiamento e conflito de interesse

\begin{tabular}{|c|c|c|c|c|c|c|c|}
\hline $\begin{array}{l}\text { Membro do grupo } \\
\text { de autores }\end{array}$ & $\begin{array}{l}\text { Local de } \\
\text { trabalho }\end{array}$ & $\begin{array}{l}\text { Verba de } \\
\text { pesquisa }\end{array}$ & $\begin{array}{c}\text { Outro apoio à } \\
\text { pesquisa ou educação } \\
\text { médica continuada }\end{array}$ & $\begin{array}{c}\text { Honorários } \\
\text { de } \\
\text { palestrantes }\end{array}$ & $\begin{array}{l}\text { Participação } \\
\text { acionária }\end{array}$ & $\begin{array}{l}\text { Consultor/ } \\
\text { conselho } \\
\text { consultivo }\end{array}$ & Outro $^{3}$ \\
\hline $\begin{array}{l}\text { Rafael Faria } \\
\text { Sanches }\end{array}$ & FMRP & - & - & $\begin{array}{c}\text { Jansen-Cilag* } \\
\text { Abbott }^{*}\end{array}$ & - & - & AstraZeneca* \\
\hline $\begin{array}{l}\text { João Mazzoncini de } \\
\text { Azevedo Marques }\end{array}$ & FMRP & - & - & - & - & - & - \\
\hline $\begin{array}{l}{ }^{*} \text { Modesto } \\
{ }^{* *} \text { Significativa } \\
\text { Nota: Fignificativa. Monta } \\
\text { Mais informações, cons }\end{array}$ & fornecic & instituição & $\begin{array}{l}\text { autor ou a colega onde } \\
\text { o. }\end{array}$ & or tem partic & ão, não dire & nte ao auto & \\
\hline
\end{tabular}

Referências

1. Ashton $\mathrm{CH}$. Adverse effects of cannabis and cannabinoids. Br J Anaesthesia. 1999;83(4):637-49.

2. Ashton CH. Pharmacology and effects of cannabis: a brief review. Br J Psychiatry. 2001;178:101-6.

3. Johns A. Psychiatric effects of cannabis. Br J Psychiatry. 2001;178:116-22.

4. Karniol IG. Cannabis sativa e derivados. In: Seibel SD, Toscano Jr A, editors. Dependência de drogas. São Paulo: Atheneu; 2001. p.131-42.

5. Hall W. The mental health effects of cannabis use. In: Gelder MG, López-Ibor JJ, Andreasen NC, editors. New Oxford textbook of psychiatry. New York: Oxford University Press; 2000. p. 550-3.

6. Nunez LA, Gurpegui M. Cannabis-induced psicosis: a cross-sectional comparison with acute schizopphrenia. Acta Psychiatr Scand. 2002;105(3):173-8.

7. Budney AJ, Hughes JR, Moore BA, Vandrey RG. A review of the validity and significance of the cannabis withdrawal syndrome. Am J Psychiatry. 2004;161(11):1967-77.

8. Leweke FM, Koethe D. Cannabis and psychiatric disorders: it is not only addiction. Addict Biol. 2008;13(2):264-75.

9. Regier DA, Farmer ME, Rae DS, Locke BZ, Keith SJ, Judd LL, Goodwin FK. Comorbidity of mental disorders with alcohol and other drug abuse. Results from the Epidemiologic Catchment Area (ECA) Study. JAMA. 1990;264(19):2511-8.

10. Kessler RC, Crum RM, Warner LA, Nelson CB, Schulenberg J, Anthony JC. Lifetime co-occurrence of DSM-III-R alcohol abuse and dependence with other psychiatric disorders in the National Comorbidity Survey. Arch Gen Psychiatry. 1997;54(4):313-21.

11. Farrell M, Howes S, Taylor C, Lewis G, Jenkins R, Bebbington P, Jarvis M, Brugha T, Gill B, Meltzer H. Substance misuse and psychiatric comorbidity: an overview of the OPCS National Psychiatric Morbidity Survey. Addict Behav. 1998;23(6):909-18.

12. Volkow ND. The reality of comorbidity: depression and drug abuse. Bio Psychiatry. 2004;56(10):714-7.

13. Strakowski SM, DelBello MP, Fleck DE, Arndt S. The impact of substance abuse on the course of bipolar disorder. Biol Psychiatry. 2000;48(6):477-85.

14. Kessler RC. The epidemiology of dual diagnosis. Biol Psychiatry. 2004;56(10):730-7.

15. Sonne SC, Brady KT, Morton WA. Substance abuse and bipolar affective disorder. J Nerv Ment Dis. 1994(6);182:349-52.

16. Feinman JA, Dunner DL. The effect of alcohol and substance abuse on the course of bipolar affective disorder. J Affective Disord. 1996;37(1):43-9.

17. Strakowski SM, DelBello MP, Fleck DE, Adler CM, Anthenelli RM, Keck PE Jr, Arnold, LM, Amicone J. Effects of co-occurring cannabis use disorders on the course of bipolar disorder after a first hospitalization for mania. Arch Gen Psychiatry. 2007;64(1):57-64.

18. Henquet C, Krabbendam L, de Graaf R, ten Have M, van Os J. Cannabis use and expression of mania in the general population. J Affect Disord. 2006;95(1-3):103-10.

19. Goldberg JF, Garno JL, Leon AC, Kocsis JH, Portera L. A history of substance abuse complicates remission from acute mania in bipolar disorder. J Clin Psychiatry. 1999;60(11):733-40.

20. van Rossum I, Boomsma M, Tenback D, Reed C, van Os J; EMBLEM Advisory Board. Does cannabis use affect treatment outcome in bipolar disorder? A longitudinal analysis. J Nerv Ment Dis. 2009;197(1):35-40.

21. DelBello MP, Hanseman D, Adler CM, Fleck DE, Strakowski SM. Twelvemonth outcome of adolescents with bipolar disorder following 
first hospitalization for a manic or mixed episode. Am J Psychiatry. 2007;164(4):582-90.

22. Brown ES, Suppes T, Adinoff B, Thomas NR. Drug abuse and bipolar disorder: comorbidity or misdiagnosis? J Affect Disord. 2001;65:105-15.

23. Cassidy F, Ahearn EP, Carroll BJ. Substance abuse in bipolar disorder. Bipolar Disord. 2001;3(4):181-8.

24. Strakowski SM, Sax KW, McElroy SL, Keck PE Jr, Hawkins JM, West SA. Psychiatric and substance abuse syndrome co-occurrence in bipolar disorder following a first psychiatric hospitalization. J Clin Psychiatry. 1998;59(9):465-71.

25. Mueser KT, Drake RE, Wallach MA. Dual diagnosis: a review of etiological theories. Addict Behav. 1998;23(6):717-34.

26. DellBello MP, Strakowski SM, Sax KW, McElroy SL, Keck PE Jr, West SA, Kmetz GF. Familial rates of affective and substance use disorders in patients with first-episode mania. J Affect Disord. 1999;56(1):55-60.

27. Fu Q, Heath AC, Bucholz KK, Nelson E, Goldberg J, Lyons MJ, True WR, Jacob T, Tsuang MT, Eisen SA. Shared genetic risk of major depression, alcohol dependence and marijuana dependence: the contribution from antisocial personality disorder in men. Arch Gen Psychiatry. 2002;59(12):1125-32.

28. Asarnow JR. Children at risk for schizophrenia: converging lines of evidence. Schizophr Bull. 1988;14(4):613-31.

29. Moeller FG, Barratt ES, Dougherty DM, Schmitz JM, Swann AC. Psychiatric aspects of impulsivity. Am J Psychiatry. 2001;158(11):1783-93.

30. Swann AC, Dougherty DM, Pazzaglia PJ, Pham M, Moeller FG. Impulsivity: a link between bipolar disorder and substance abuse. Bipolar Disord. 2004;6(3):204-12.

31. Sasson Y, Chopra M, Harrari E, Amitai K, Zohar J. Bipolar comorbidity: from diagnostic dilemmas to therapeutic challenge. Int J Neuropsychopharmacol. 2003;6(2):139-44.

32. Ribeiro M, Laranjeira R, Cividanes G. Bipolar disorders and substance abuse. Rev Psiq Clin. 2005;32(Supl 1):78-88

33. Merikangas KR, Herrell R, Swendsen J, Rössler W, Ajdacic-Gross V, Angst J. Specificity of bipolar spectrum conditions in the comorbidity of mood and substance use disorders: results from the Zurich cohort study. Arch Gen Psychiatry. 2008;65(1):47-52.

34. Levin FR, Hennessy G. Bipolar disorder and substance abuse. Biol Psychiatry. 2004;56(10):738-48.

35. Ashton CH, Moore PB, Gallagher P, Young AH. Cannabinoids in bipolar affective disorder: a review and discussion of their therapeutic potential. J Psychopharmacol. 2005;19(3):293-300.

36. Degenhardt L, Hall W, Lynskey M, Coffey C, Patton G. The association between cannabis use and depression: a review of the evidence. In: Castle D, Murray R, editors. Marijuana and madness. Cambridge: Cambridge University Press; 2004. p. 54-74.

37. Mathew RJ, Wilson WH, Tant SR. Acute changes in cerebral blood flow associated with marijuana smoking. Acta Psychiatr Scand. 1989;79(2):118-28.

38. D'Souza DC, Abi-Saab WM, Madonick S, Forselius-Bielen K, Doersch A, Braley G, Gueorguieva R, Cooper TB, Krystal JH. Delta-9tetrahydrocannabinol effects in schizophrenia: implications for cognition, psychosis, and addiction. Biol Psychiatry. 2005;57(6):594-608.

39. Murray RM, Sham P, Van Os J, Zanelli J, Cannon M, McDonald C. A developmental model for similarities and dissimilarities between schizophrenia and bipolar disorder. Schizophr Res. 2004;71(2-3):405-16.

40. Michael N, Erfurth A, Ohrmann P, Gossling M, Arolt V, Heindel W, Pfleiderer B. Acute mania is accompanied by elevated glutamate/glutamine levels within the left dorsolateral prefrontal cortex. Psychopharmacology (Berl). 2003;168(3):344-6.

41. Ketter TA, Wang PW The emerging differential roles of GABAergic and antiglutamatergic agents in bipolar disorders. J Clin Psychiatry. 2003;64(Suppl 3):15-20.

42. Friedman SD, Dager SR, Parow A, Hirashima F, Demopulos C, Stoll AL, Lyoo IK, Dunner DL, Renshaw PF. Lithium and valproic acid treatment effects on brain chemistry in bipolar disorder. Biol Psychiatry. 2004;56(5):340-8.

43. Tomasini MC, Ferraro L, Bebe BW, Tanganelli S, Cassano T, Cuomo V, Antonelli T. Delta(9)-tetrahydrocannabinol increases endogenous extracellular glutamate levels in primary cultures of rat cerebral cortex neurons: involvement of CB(1) receptors. J Neurosci Res. 2002;68(4): 449-53.

44. Post RM, Rubinow DR, Ballenger JC. Conditioning, sensitization, and kindling: Implications for the course of affective illness. In: Post RM,
Ballenger JC, editors. The neurobiology of mood disorders. Baltimore: Williams \& Wilkins; 1984; p.432-66.

45. Goodwin FK, Jamison KR. Manic depressive illness. New York: Oxford University Press; 1990.

46. Lieberman JA, Kinon BJ, Loebel AD. Dopaminergic mechanisms in idiopathic and drug induced psychoses. Schizophr Bull. 1990;16(1):97-110.

47. Bovasso GB. Cannabis abuse as a risk factor for depressive symptoms. Am J Psychiatry. 2001;158(12):2033-7.

48. van Laar M, van Dorsselaer S, Monshouwer K, de Graaf R. Does cannabis use predict the first incidence of mood and anxiety disorders in the adult population? Addiction. 2007;102(8):1251-60.

49. Carroll KM. Behavioral therapies for co-occuring substance use and mood disorders. Biol Psychiatry. 2004;56(10):778-84

50. Ridgley MS, Johson S. Drug and alcohol services. In: Thornicroft G, Szmukler G, editors. Textbook of community psychiatry. Devon: Oxford University Press; 2001. p.347-67.

51. Raimo EB, Schuckit MA. Alcohol dependence and mood disorders. Addict Behav. 1998;23(6):933-46.

52. Sanches RF, Assunção S, Hetem LA. Impacto da comorbidade no diagnóstico e tratamento do transtorno bipolar. Rev Psiq Clin. 2005;32(Suppl.1):71-7.

53. Weiss RD, Greenfield SF, Najavits LM, Soto JA, Wyner D, Tohen M, Griffin ML. Medication compliance among patients with bipolar disorder and substance use disorder. J Clin Psychiatry. 1998;59(4):172-4.

54. Potvin S, Stip E, Roy JY. The effect of quetiapine on cannabis use in 8 psychosis patients with drug dependency. Can J Psychiatry. 2004;49(10):711-20.

55. Grinspoon L, Bakalar JB. The use of cannabis as a mood stabilizer in bipolar disorder: anecdotal evidence and the need for clinical research. J Psychoactive Drugs. 1998;30(2):171-7.

56. Gruber AJ, Pope HG Jr, Brown ME. Do patients use marijuana as an antidepressant? Depression. 1996;4(2):77-80.

57. Regelson W, Butler JR, Schulz J, Kirk T, Peek L, Green ML, Zalis MO. Delta-9-THC as an effective antidepressant and appetitestimulating agent in advanced cancer patients. In Braude M C, Szara S, editors. The pharmacology of marihuana. New York: Raven Press; 1976. p.763-76.

58. Goodwin G, Sachs G. Definitions: Diagnosis and comorbidity. Bipolar disorder - fast facts. Oxford: Health Press; 2004.

59. Zuardi AW, Crippa JA, Hallak JE, Moreira FA, Guimarāes FS. Cannabidiol, a Cannabis sativa constituent, as an antipsychotic drug. BrazJ Med Biol Res. 2006;39(4):421-9.

60. Porter R, Ferrier N, Ashton H. Anticonvulsants as mood stabilisers. Adv Psychiatr Treat. 1999;5:96-103.

61. Ashton $\mathrm{H}$, Young AH. GABA-ergic drugs: exit stage left, enter stage right. $J$ Psychopharmacol. 2003;17(2):174-8.

62. Valvassori SS, Elias G, de Souza B, Petronilho F, Dal-Pizzol F, Kapczinski F, Trzesniak C, Tumas V, Dursun S, Chagas MH, Hallak JE, Zuardi AW, Quevedo J, Crippa JA. Effects of cannabidiol on amphetamineinduced oxidative stress generation in an animal model of mania. $J$ Psychopharmacol. In press 2009.

63. Zuardi A, Crippa J, Dursun S, Morais S, Vilela J, Sanches R, Hallak J. Cannabidiol was ineffective for manic episode of bipolar affective disorder. J Psychopharmacol. 2010;24(1):135-7.

64. Compton WM, Cottler LB, Jacobs JL, Ben-Abdallah A, Spitznagel El. The role of psychiatric disorders in predicting drug dependence outcomes. Am J Psychiatry. 2003;160(5):890-5.

65. Grant BF. Comorbidity between DSM-IV drug use disorders and major depression: results of a national survey of adults. J Subst Abuse. 1995;7(4):481-97.

66. Chen CY, Wagner FA, Anthony JC. Marijuana use and the risk of Major Depressive Episode. Epidemiological Evidence from the United States National Comorbidity Survey. Soc Psychiarty Psychiatr Epidemiol. 2002;37(5):199-206.

67. Degenhardt L, Hall W, Lynskey M. The relationship between cannabis use, depression and anxiety among Australian adults: findings from the National Survey of Mental Health and Well-Being. Soc Psychiarty Psychiatr Epidemiol. 2001;36(5):219-27.

68. Rey JM, Sawyer MG, Raphael B, Patton GC, Lynskey M. Mental health of teenagers who use cannabis: results of an Australian survey. Br J Psychiatry. 2002;180:216-21.

69. Fergusson DM, Horwood LJ, Swain-Campbell N. Cannabis use and psychosocial adjustment in adolescent and young adulthood. Addiction. 2002;97(9):1123-35 
70. Angst J. Comorbidity of mood disorders: a longitudinal prospective study. $\mathrm{Br}$ J Psychiatry Suppl. 1996;30:31-7.

71. Rey JM, Tennant CC. Cannabis and mental health. BMJ. 2002;325(7374):11834.

72. Macleod J, Oakes R, Copello A, Crome I, Egger M, Hickman M, Oppenkowski T, Stokes-Lampard H, Davey Smith G. Psychological and social sequelae of cannabis and other illicit drug use by young people: a systematic review of longitudinal, general population studies. Lancet. 2004;363(9421):1579-88.

73. Macleod J, Hickman M. Commentary: understanding pathways to cannabis use and from use to harm. Int J Epidemiol. 2006;35(3):680-2.

74. Kandel D, Chen K. Types of marijuana users by longitudinal course. J Studies Alcohol. 2000;61(3):367-78.

75. Hofstra M, van der Ende J, Verhultz F. Child and adolescent problems predict DSM-IV disorders in adulthood: a 14-year follow-up of a dutch epidemiological sample. J Am Acad Child Adolescent Psychiatry. 2002;41(2):182-9.

76. Moore TH, Zammit S, Lingford-Hughes A, Barnes TR, Jones PB, Burke M, Lewis G. Cannabis use and risk of psychotic or affective mental health outcomes: a systematic review. Lancet. 2007;370(9584):319-28.

77. Arseneault L, Cannon M, Poulton R, Murray R, Caspi A, Moffi tt TE. Cannabis use in adolescence and risk for adult psychosis: longitudinal prospective study. BMJ. 2002;325(7374):1212-3.

78. Brook DW, Brook JS, Zhang C, Cohen P, Whiteman M. Drug use and the risk of major depressive disorder, alcohol dependence and substance use disorders. Arch Gen Psychiatry. 2002;59(11):1039-44.

79. Patton GC, Coffey C, Carlin JB, Degenhardt L, Lynskey M, Hall W. Cannabis use and mental health in young people: cohort study. BMJ. 2002;325(7374):1195-8.

80. Borowsky IW, Ireland M, Resnick MD. Adolescent suicide attempts: risks and protectors. Pediatrics. 2001;107(3):485-93.

81. Brook JS, Rosen Z, Brook DW. The effect of early marijuana use on later anxiety and depressive symptoms. New York: NYS Psychologist; 2001. p.35-40.

82. Wade TJ, Pevalin DJ. Adolescent delinquency and health. Can J Criminol Crim Just. 2005; 47:619-54.

83. Beard JR, Dietrich UC, Brooks LO, Brooks RT, Heathcote K, Kelly B. Incidence and outcomes of mental disorders in a regional population: the Northern Rivers Mental Health Study. Aust N Z J Psychiatry. 2006;40(8):674-82.

84. Harder VS, Morral AR, Arkes J. Marijuana use and depression among adults: Testing for causal associations. Addiction. 2006;101(10):1463-72.

85. Harder VS, Stuart EA, Anthony JC. Adolescent cannabis problems and young adult depression: male-female stratified propensity score analyses. $\mathrm{Am} \mathrm{J}$ Epidemiol. 2008;168(6):592-601.

86. Marihuana Treatment Project Research Group. Brief treatments for cannabis dependence: findings from a mutisite trial. J Consult Clin Psychol. 2004;72(3):455-66.

87. Riggs PD, Mikulich-Gilbertson SK, Davies RD, Lohman M, Klein C, Stover SK. A randomized controlled trial of fluoxetine and cognitive behavioral therapy in adolescents with major depression, behavior problems, and substance use disorders. Arch Pediatr Adolesc Med. 2007;161(11):102634.

88. Bhattacharyya S, Crippa JA, Martin-Santos R, Winton-Brown T, FusarPoli P. Imaging the neural effects of cannabinoids: current status and future opportunities for psychopharmacology. Curr Pharm Des. 2009;15(22):2603-14.

89. Crippa JA, Zuardi AW, Martín-Santos R, Bhattacharyya S, Atakan Z, McGuire P, Fusar-Poli P. Cannabis and anxiety: a critical review of the evidence. Hum Psychopharmacol. 2009;24(7):515-23. 